\title{
Abscopal effect of radiation on lymph node metastasis in esophageal carcinoma: A case report and literature review
}

\author{
XIANGFEI ZHAO, JINGBO KANG and RUGANG ZHAO \\ Department of Medical Oncology, Navy General Hospital, Beijing 100048, P.R. China
}

Received February 11, 2018; Accepted June 28, 2018

DOI: $10.3892 / \mathrm{ol} .2018 .9084$

\begin{abstract}
The Abscopal effect is a rare phenomenon observed in the treatment of metastatic cancer, where localized irradiation causes a response in non-irradiated tumor sites. Due to the recent success of immunotherapies, the Abscopal effect of radiation therapy has received renewed clinical interest. However, there is limited knowledge regarding the Abscopal effect and radiotherapy treatment of patients with esophageal carcinoma. The present study reports the case of a 65 -year-old male patient, who presented with esophageal carcinoma and lymph node metastasis. A transthoracic esophagectomy with left cervical, mediastinal and abdominal lymphadenectomies was performed. A total of 4 cycles of chemotherapy and maintenance therapy with Pembrolizumab was performed until September 2016. Metastases in the left retroperitoneal lymph node in addition to extensive metastases to the pelvic lymph node were observed. The patient received Cyberknife radiotherapy with a dose of 42 Gy in 6 daily fractions targeted at the left retroperitoneal lymph node. Two months after radiation therapy, a positron emission tomography-computed tomography scan revealed complete regression of all lymph node metastases. There is increasing clinical evidence supporting the efficacy of the Abscopal effect, which may be initiated by high-dose radiation. Further research is required to make the Abscopal effect clinically relevant, however it may have potential as a treatment option.
\end{abstract}

\section{Introduction}

Radiotherapy (RT) is the generally prescribed treatment for localized cancer $(1,2)$. However, evidence from clinical and experimental data has suggested that there may be tumor regression at non-irradiated tumor sites in addition to at the irradiated site (so called abscopal effects) (3-5). The Abscopal

Correspondence to: Dr Xiangfei Zhao, Department of Medical Oncology, Navy General Hospital, 6 Fu Cheng Road, Beijing 100048, P.R. China

E-mail: article1977@163.com

Key words: abscopal effect, esophageal carcinoma, radiotherapy, immune checkpoint inhibitors, lymph node metastasis effect is a rare phenomenon, which is probably associated with an immune effect triggered by a high-dose of radiation. The biological mechanism underlying this effect remain to be elucidated. An potential mechanism involved in radiation-induced immune response which can convert immunologically 'cold' tumors into 'hot' tumors. Including the upregulation of immunogenic surface molecules after RT and antigen processing and Modulation the tumor microenvironment via secreted factors $(6,7)$. The combination of radiation and immunotherapy might be a useful strategy for the treatment of malignant tumors. Esophageal cancer has been ranked as the sixth leading cause of cancer-related death over the world (8). Radiation is the main treatment for esophageal cancer (8). Some clinical trials to evaluate immunotherapy in combination with radiation are currently underway (NCT02476123 and UMIN000021480). Here, we describe a case of a gentleman with esophageal carcinoma and lymph node metastasis, who received only focal radiation treatment to one metastastic lymph node, and complete and sustained radiological regression of other metastases.

\section{Case report}

In late March 2016, a 65-year-old male patient presented with esophageal carcinoma and lymph node metastasis. The patient had an upper gastrointestinal endoscopy screening, which revealed an esophageal lesion $25 \mathrm{~cm}$ from the incisors. A transthoracic esophagectomy with left cervical, mediastinal, and abdominal lymphadenectomies was carried out. The results of the biopsy showed undifferentiated invasive squamous cell carcinoma and multiple lymph node metastases. A follow-up positron emission tomography (PET) scan showed a fluorodeoxyglucose avid lesion at the retroperitoneal lymph nodes (Fig. 1). The patient was given an intravenous chemotherapy regimen of three weekly doses of cisplatin $\left(80 \mathrm{mg} / \mathrm{m}^{2}\right)$ and docetaxel $\left(75 \mathrm{mg} / \mathrm{m}^{2}\right)$. A subsequent PET-CT scan indicated a complete response without tumor activity after 1 month (Fig. 2).

The patient received a total of 4 cycles of chemotherapy until August 2016. Subsequently, maintenance therapy was administered. Pembrolizumab was given at $2 \mathrm{mg} / \mathrm{kg}$ on day 1 of every 2-week cycle. In September 2016, lymph node metastases were detected again. A PET-CT scan showed apparent metastases of the left retroperitoneal lymph node in addition to extensive metastases in the pelvic lymph node (Fig. 3). The patient reported feeling weak and could not tolerate 


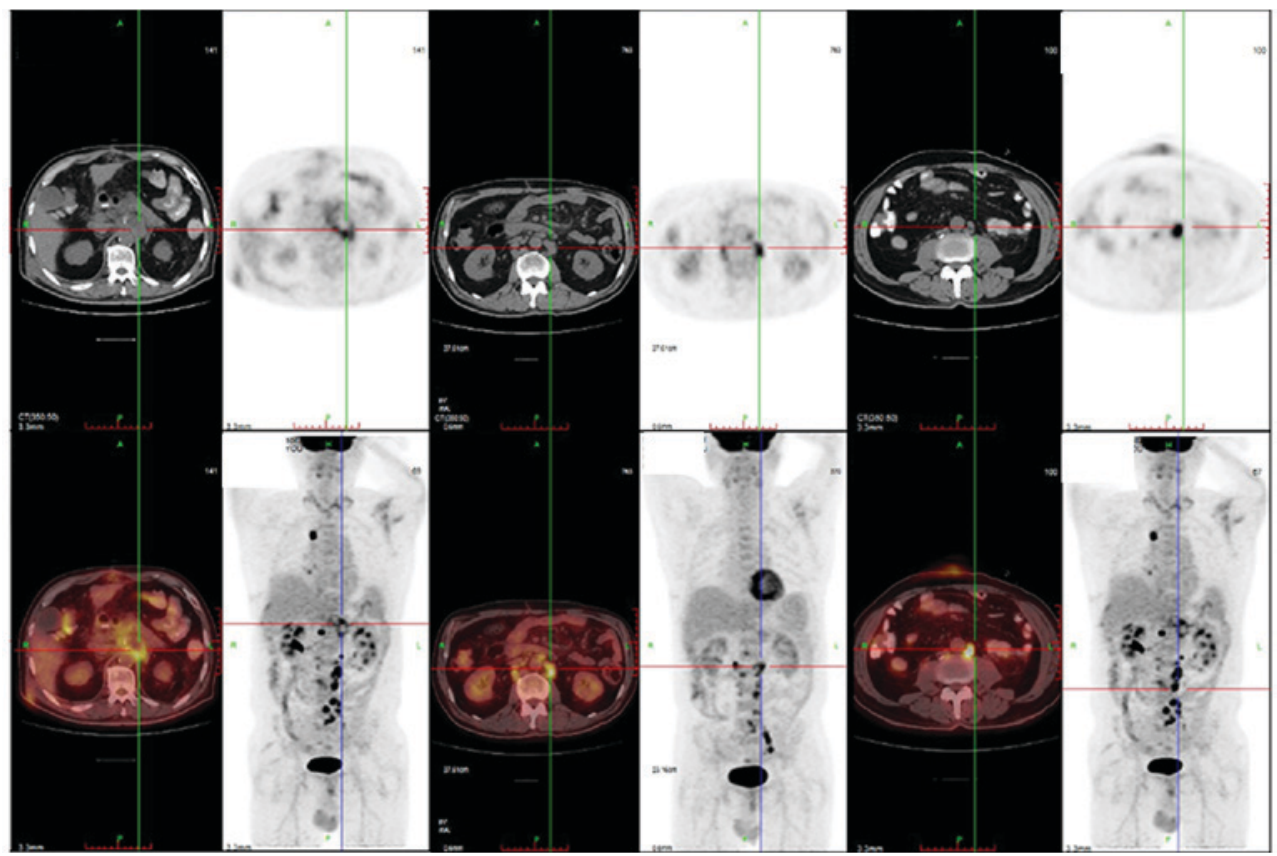

Figure 1. Positron emission tomography scan of retroperitoneal lymph node metastasis following an operation.

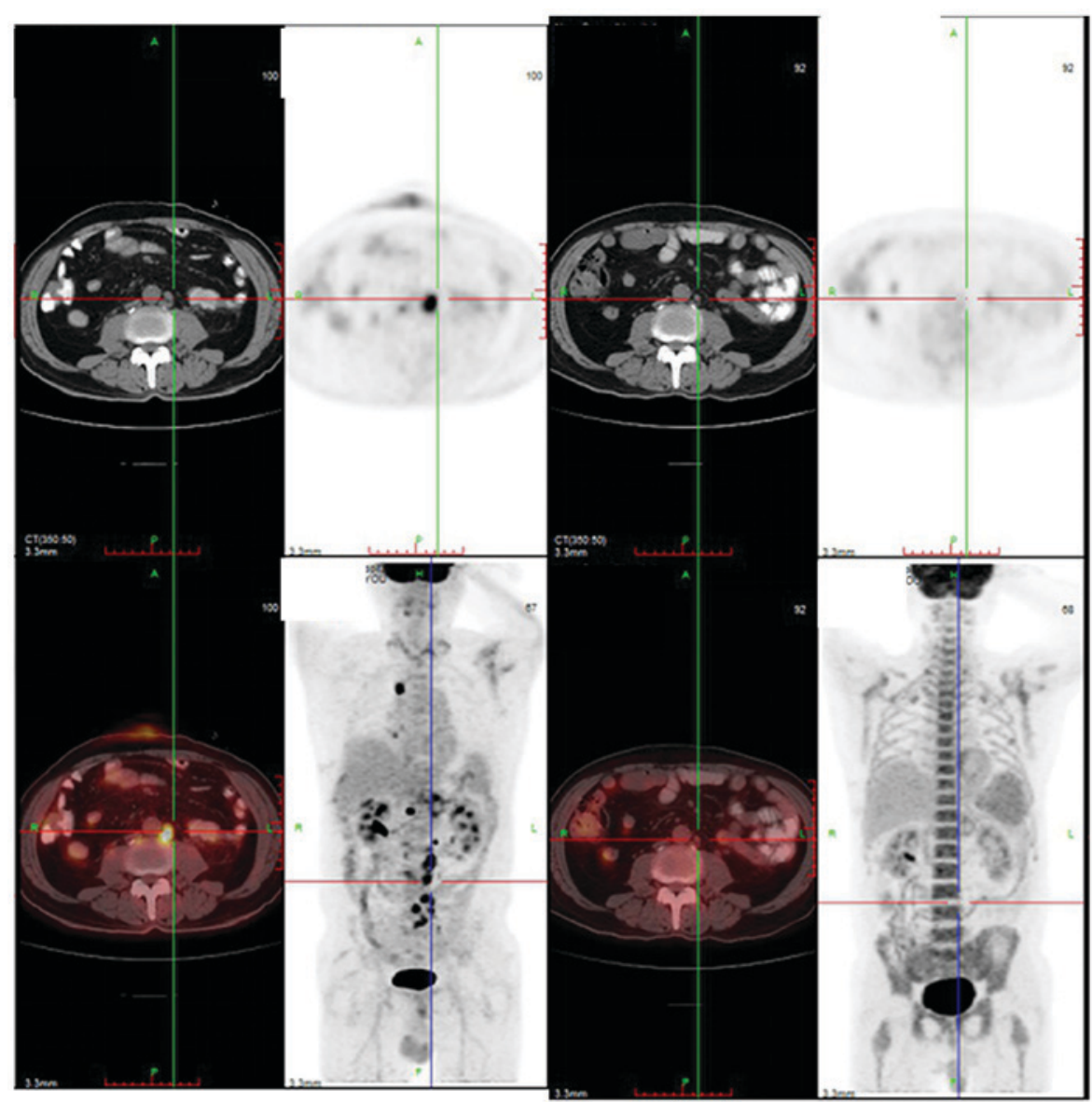

Figure 2. Positron emission tomography scans showing a complete remission of the lymph node metastasis prior to second chemotherapy.

chemotherapy. The patient received Cyberknife with a dose of 42 Gy in 6 daily fractions (Fig. 4). Two months after radiation therapy, a PET-CT scan showed, surprisingly, a complete regression of the lymph node metastases (Figs. 5 and 6).
At the same time, peripheral blood samples were collected during treatment for metastases detection. The changes in TP53 and RB1 mutations were used to quantify the circulating tumor DNA (ctDNA) using Bio-Rad QX200 


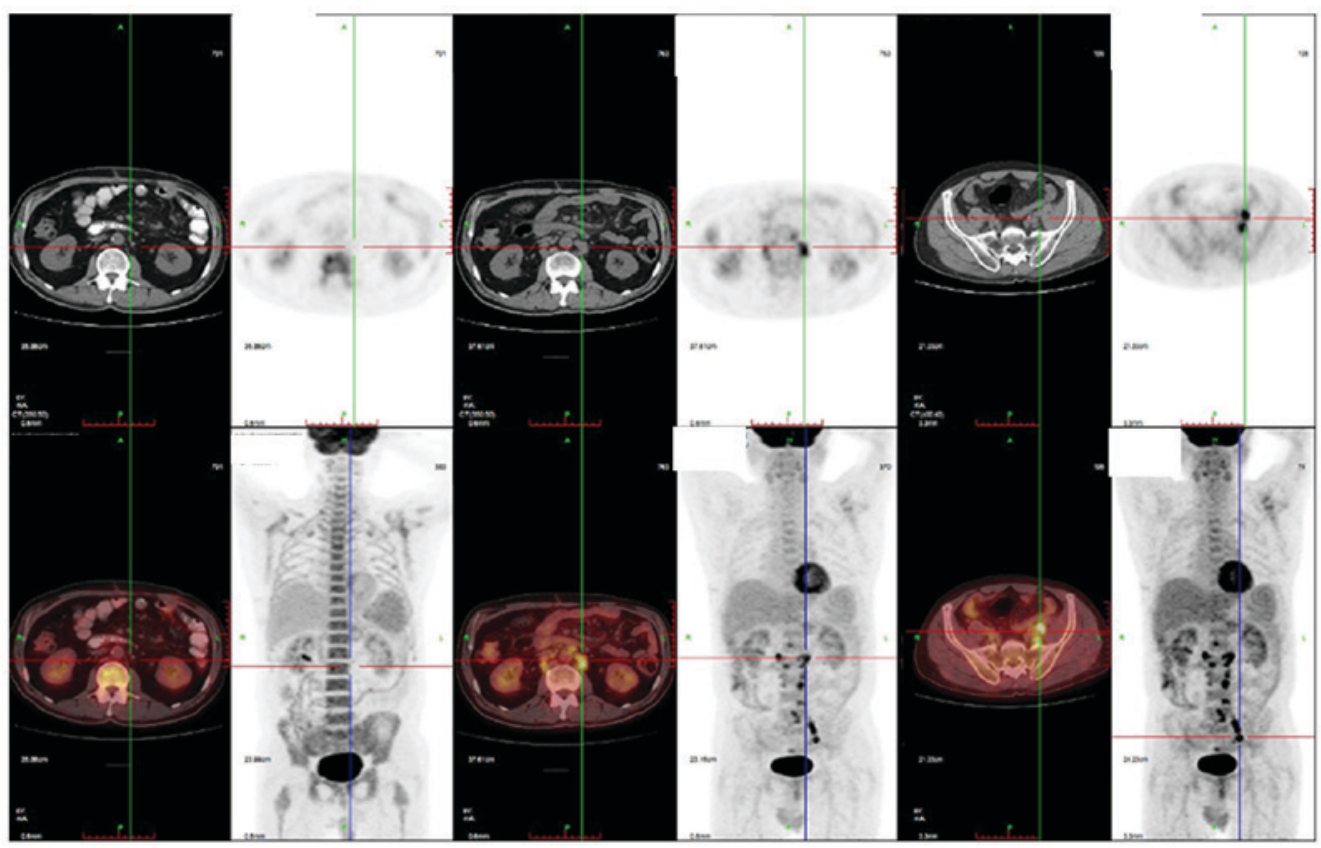

Figure 3. Positron emission tomography scans showing left retroperitoneal lymph node and pelvic lymph node metastasis during maintenance therapy with Pembrolizumab.

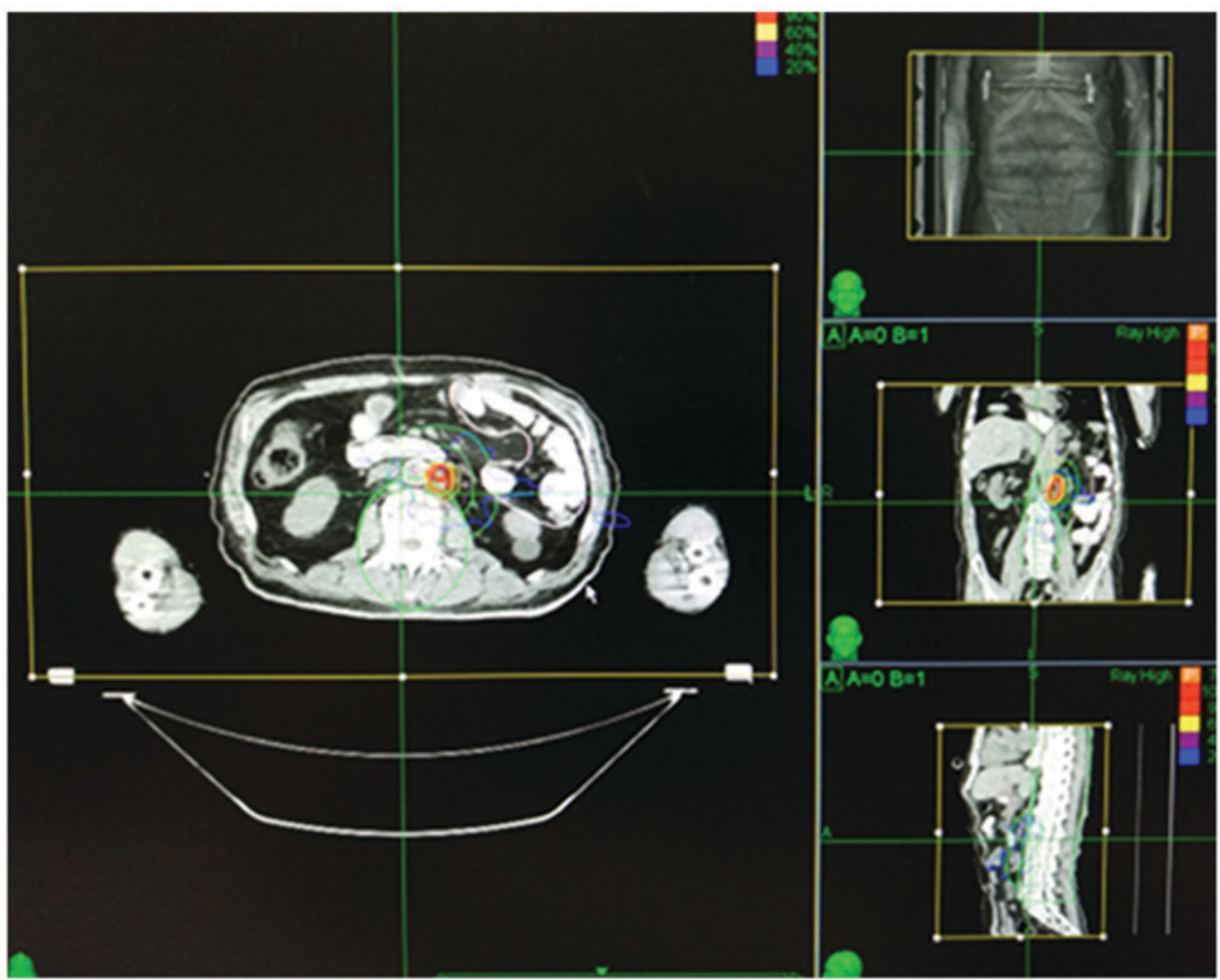

Figure 4. Radiation dosimetry plan for the lymph nodes.

droplet digital PCR system during treatment with radiation and Pembrolizumab. Mutant allele concentration (copies $/ \mu 1$, CMUT) and wild-type allele concentration (copies/ $\mu$ l, CWT) were calculated in the test. The primers and probes were synthesized by Geneseeq Biotech (NanJing, China). The TP53 and RB1 mutation was evaluated by mutant allele frequency (MAF). MAF was calculated as: $\mathrm{MAF}=\mathrm{CMUT} /(\mathrm{CMUT}+$
CWT). Approximately 49 days after the start of the combination therapy, abundances of TP53 mutation dropped from 13-4\%, abundances of RB1 mutation dropped from 9-1\%. Between November 2016 to June 2017, the patient underwent maintenance therapy with Pembrolizumab and the disease remained stable with no imaging evidence of disease recurrence. 


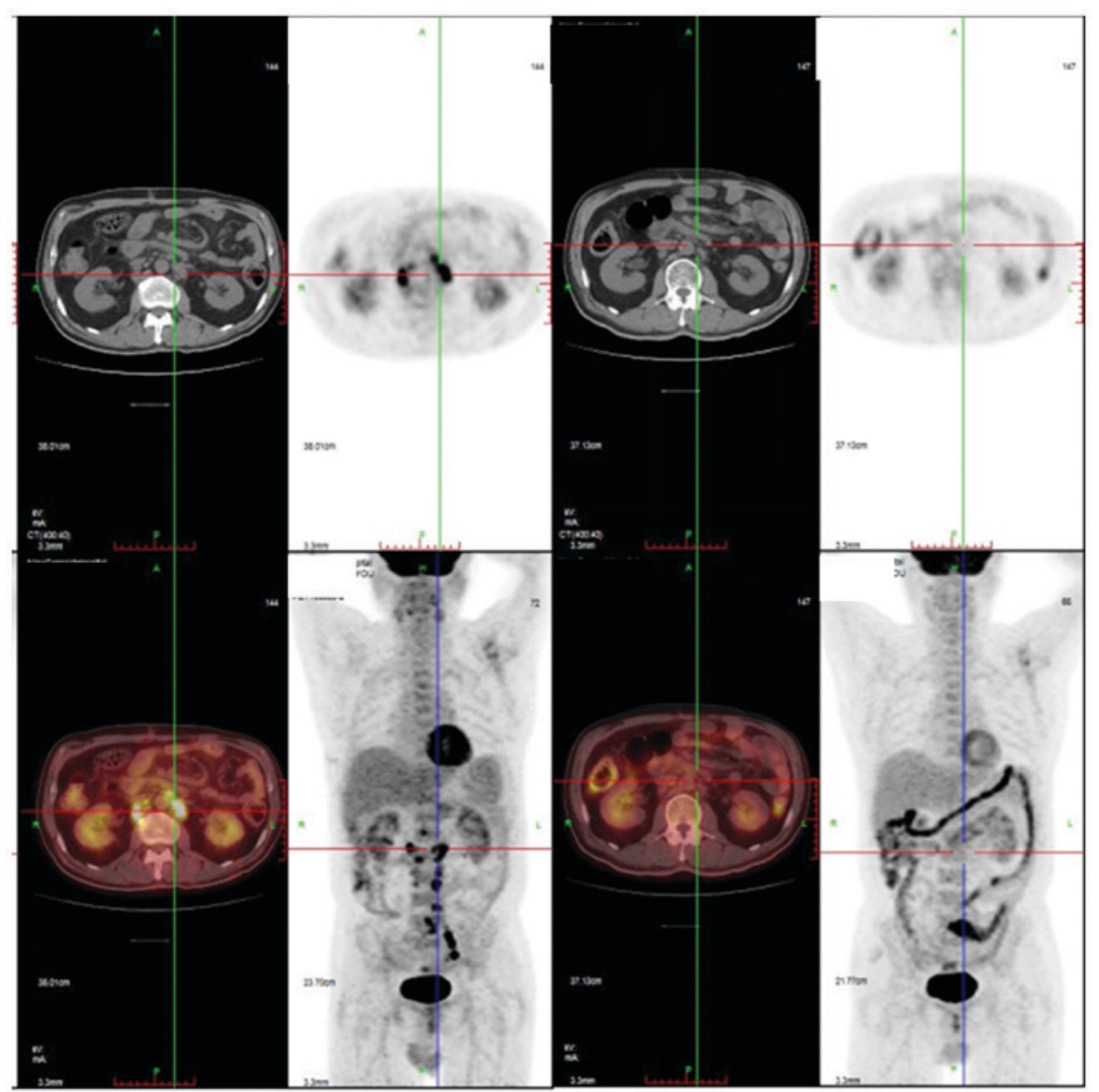

Figure 5. Positron emission tomography scans showing a complete regression of the lymph node metastasis at 2 month following Cyberknife.

\section{Discussion}

It has been demonstrated that immunotherapies with PD-1 checkpoint inhibitors may be a potent new therapeutic option in malignant neoplasms. Kojima et al reported data on nivolumab for patients with advanced eosophageal squamous cell carcinoma (ESCC) who were not preselected by PD-L1 status. The median overall survival was 12.1 months in the 64 evaluable patients and PR rate and CR rate were 15.6 and $1.6 \%$, respectively. There were no treatment-related deaths (9). Another result from KEYNOTE-028 indicated that 52.2\% of patients with advanced esophageal carcinoma that were treated by Pembrolizumab showed some degree of tumor shrinkage and had manageable side effects (10).

In a clinical setting, despite being rare, the benefit of a combination of immune checkpoint inhibitors and RT has also been observed. Our patient presented with regression of the non-irradiated lymph node metastases after treatment with stereotactic ablative radiotherapy (SABR). This case is an example of the so-called 'Abscopal effect'. The phenomenon of the Abscopal effect was first described by R.H. Mole in 1953 (11). With the recent success of immunotherapies, using the Abscopal effect of RT has garnered renewed clinical interest however, there is limited knowledge about the Abscopal effect and RT in the treatment of patients with esophageal carcinoma. However, RT alone is insufficient to induce the Abscopal effect. Some preclinical experiments have shown that checkpoint inhibitors could be radiosensitizing therapies that cause at least local tumor control (12). In a case report, a patient with metastatic non-small cell lung cancer (NSCLC) who started on ipilimumab plus RT for a liver metastasis had a striking systemic response (13). Of note, Grimaldi reported a study enrolling 21 patients with advanced melanoma who emerge disease progressed after receiving the immunoadjuvant ipilimumab (anti-CTLA4) and then RT for cranial or extracranial sites was executed, which resulted in an Abscopal response in 11 patients $(52 \%)$, median overall survival was superior in patients that exhibited the Abscopal effect compared with nonresponders (14). In another study, a clinical case series using a stereotactic radiation regimen in 16 patients, including 12 cases of metastatic melanoma, 2 metastatic NSCLC, and 2 metastatic renal cell carcinoma was conducted. Only three patients with melanoma developed an Abscopal effect; $18.7 \%$ of the patients involved (15). Yazan Abuodeh reviewed a total of 46 cases of the Abscopal effect that had been identified from 1969 to 2014 with a median radiation dose of $31 \mathrm{~Gy}$, median follow-up time of 17.5 months, and the median documented time to notice the Abscopal effect was 2 months (5).

It is unclear about which dose/fractionation regimens optimally enhance the antitumor immune response of RT combined with immunomodulation. However some clinical studies have investigated this. A phase I/II clinical study of 34 patients with metastatic castration-resistant prostate cancer were treated with ipilimumab and 8 Gy fractions in bone metastases. A complete response was reported in one patient, stable disease in six patients, and prostate-specific antigen decreases 


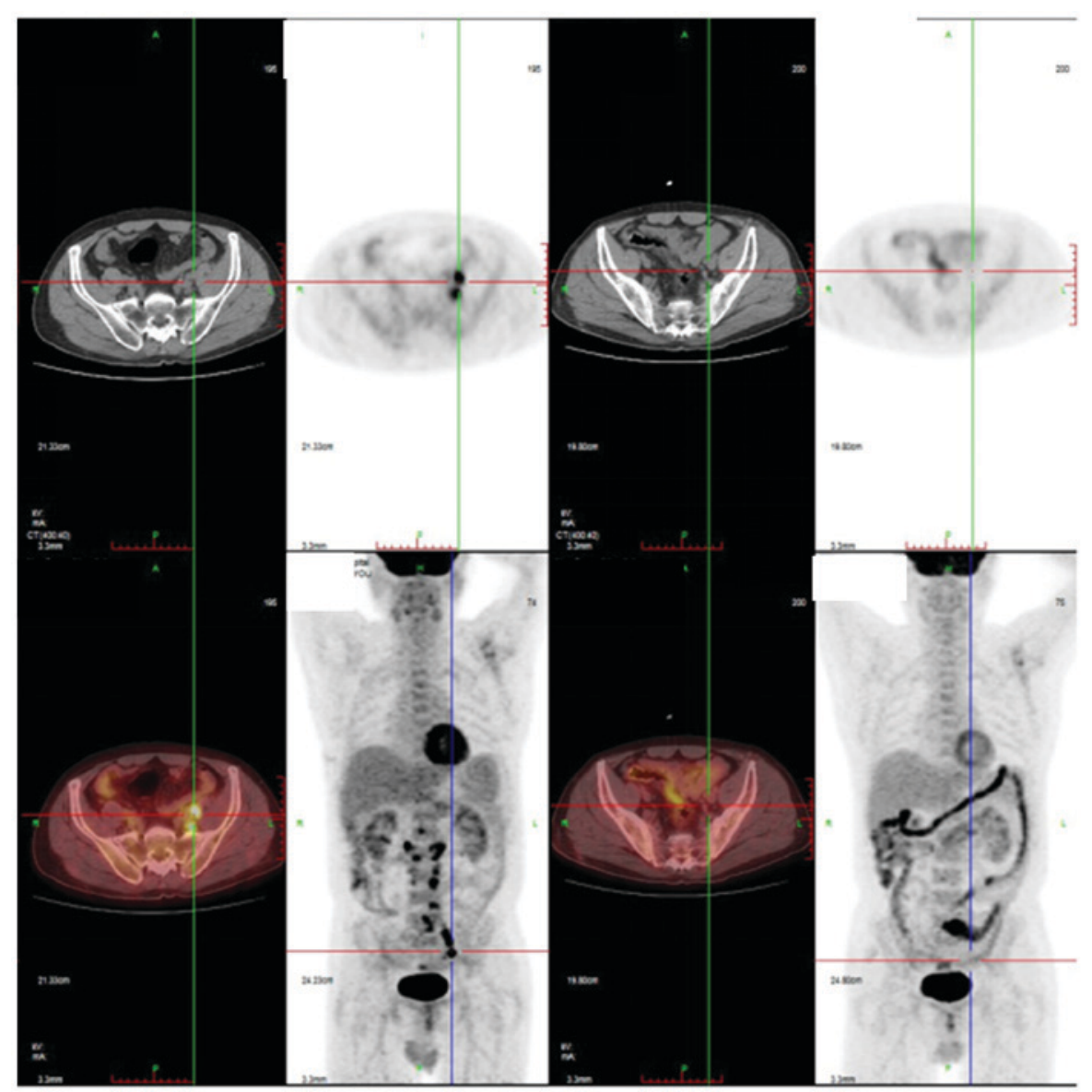

Figure 6. The abscopal response of the pelvic lymph node.

of $\geq 50 \%$ were recorded (16). Raffaella Marconi reviewed the Abscopal effect in preclinical models. SABR treatments with a high biological equivalent dose (BED) could be considered to be an effective strategy in triggering the Abscopal effect. In particular, while a BED of $60 \mathrm{~Gy}$ is applied the probability of stimulating the Abscopal effect is $50 \%$. The article indicted the hypothesis that the occurrence rate of Abscopal effects in preclinical models increaseing with BED (17).

In this case study, ctDNA was used to monitor the tumor burden. Recent clinical results reported on the use of cell-free DNA to monitor tumor burden during anti-PD1 immunotherapy. L. Cabel reported that ctDNA was measured at the baseline in 10 out of 15 patients with non-small cell lung cancer, uveal melanoma or microsatellite-unstable colorectal cancer who were treated by nivolumab or pembrolizumab monotherapy. Baseline ctDNA was a significant prognostic factor in terms of progression-free survival (18). In another study, Nicolas Guibert monitored the level of KRAS-mutated ctDNA in serial plasma samples from two patients with adenocarcinoma who had experienced pseudo-progression. ctDNA showed rapid and dramatic decreases in the pseudo-progressive patients, whereas it was strongly increased in the progressive patient (19).

We have reported a case of the Abscopal effect treated with cyberknife RT until lymph node metastasis regression occurred in the unirradiated lymph node in esophageal carcinoma. There is increasing clinical evidence supporting the efficacy of the Abscopal effect initiated by high-dose radiation treatment. Further research to make the Abscopal effect clinically relevant is worth exploring.

\section{Acknowledgements}

Not applicable.

\section{Funding}

No funding was received.

\section{Availability of data and materials}

All data generated or analyzed during this study are included in this published article.

\section{Authors' contributions}

ZXF was the project leader and collected and analysed the data. ZRG was the physician who handled the case. KJB analyzed and interpreted PET/CT images. All authors read and approved the final manuscript.

\section{Ethics approval and consent to participate}

Written informed consent was obtained from the patient and the present study was approved by the Ethics Board of the Navy General Hospital (Beijing, China). 


\section{Patient consent for publication}

Written informed consent was obtained from the patient for the publication of their data.

\section{Competing interests}

The authors declare that they have no competing interests.

\section{References}

1. Orth M, Lauber K, Niyazi M, Friedl AA, Li M, Maihöfer C, Schüttrumpf L, Ernst A, Niemöller OM and Belka C: Current concepts in clinical radiation oncology. Radiat Environ Biophys 53: 1-29, 2014.

2. Hoskin PJ and Bhattacharya IS: Protons and more: State of the art in radiotherapy. Clin Med (Lond) 14 (Suppl 6): s61-s65, 2014

3. Xie G, Gu D, Zhang L, Chen S and Wu D: A rapid and systemic complete response to stereotactic body radiation therapy and pembrolizumab in a patient with metastatic renal cell carcinoma. Cancer Biol Ther 18: 547-551, 2017.

4. Sharabi A, Kim SS, Kato S, Sanders PD, Patel SP, Sanghvi P, Weihe E and Kurzrock R: Exceptional response to nivolumab and stereotactic body radiation therapy (SBRT) in neuroendocrine cervical carcinoma with high tumor mutational burden: Management considerations from the center for personalized cancer therapy at UC San diego moores cancer center. Oncologist 22: 631-637, 2017.

5. Abuodeh Y, Venkat P and Kim S: Systematic review of case reports on the abscopal effect. Cur Probl Cancer 40: 25-37, 2016

6. Garnett C, Palena C, Chakarborty M, Tsang KY, Schlom J and Hodge JW: Sublethal irradiation of human tumor cells modulates phenotype resulting in enhanced killing by cytotoxic T lymphocytes. Cancer Res 64: 7985-7994, 2004.

7. Coppé JP, Patil CK, Rodier F, Sun Y, Muñoz DP, Goldstein J, Nelson PS, Desprez PY and Campisi J: Senescence-associated secretory phenotypes reveal cell-nonautonomous functions of oncogenic RAS and the p53 tumor suppressor. PLoS Biol 6: 2853-2868, 2008.

8. Ferlay J, Soerjomataram I, Dikshit R, Eser S, Mathers C, Rebelo M, Parkin DM, Forman D and Bray F: Cancer incidence and mortality worldwide: Sources, methods and major patterns in GLOBOCAN 2012. Int J Cancer 136: E359-E386, 2015.

9. Kojima T, Hara H, Yamaguchi K, Hironaka S, Iwasa $S$, Kato K, Iwasa S, Kato K, Tsushima T, Yasui T, et al: Phase II study of nivolumab (ONO-4538/BMS-936558) in patients with esophageal cancer: Preliminary report of overall survival. J Clin Oncol 34 (Suppl 4): TPS175-TPS175, 2016.
10. Doi T, Piha-Paul SA, Jalal SI, Mai-Dang H, Yuan S, Koshiji M, Csiki I and Bennouna J: Pembrolizumab (MK-3475) for patients (pts) with advanced esophageal carcinoma: Preliminary results from KEYNOTE-028. J Clin Oncol 33 (15-Suppl): S4010, 2015.

11. Mole RH: Whole body irradiation; radiobiology or medicine? $\mathrm{Br}$ J Radiol 26: 234-241, 1953.

12. Polistena A, Johnson LB, Ohiami-Masseron S, Wittgren L, Bäck S, Thornberg C, Gadaleanu V, Adawi D and Jeppsson B: Local radiotherapy of exposed murine small bowel: Apoptosis and inflammation. BMC Surg 8: 1, 2008.

13. Golden EB, Demaria S, Schiff PB, Chachoua A and Formenti SC: An abscopal response to radiation and ipilimumab in a patient with metastatic non-small cell lung cancer. Cancer Immunol Res 1: 365-372, 2013.

14. Grimaldi AM, Simeone E, Giannarelli D, Muto P, Falivene S, Borzillo V, Giugliano FM, Sandomenico F, Petrillo A, Curvietto M, et al: Abscopal effects of radiotherapy on advanced melanoma patients who progressed after ipilimumab immunotherapy. Oncoimmunology 3: e28780, 2014.

15. Ribeiro Gomes J, Schmerling RA, Haddad CK, Racy DJ, Ferrigno R, Gil E, Zanuncio P and Buzaid AC: Analysis of the abscopal effect with anti-PD1 therapy in patients with metastatic solid tumors. J Immunother 39: 367-372, 2016

16. Slovin SF, Higano CS, Hamid O, Tejwani S, Harzstark A, Alumkal JJ, Scher HI, Chin K, Gagnier P, McHenry MB and Beer TM: Ipilimumab alone or in combination with radiotherapy in metastatic castration-resistant prostate cancer: Results from an open-label, multicenter phase I/II study. Ann Oncol 24: 1813-1821, 2013

17. Marconi R, Strolin S, Bossi G and Strigari L: A meta-analysis of the abscopal effect in preclinical models: Is the biologically effective dose a relevant physical trigger? PLoS One 12: e0171559, 2017.

18. Cabel L, Riva F, Servois V, Livartowski A, Daniel C, Rampanou A, Lantz O, Romano E, Milder M, Buecher B, et al: Circulating tumor DNA changes for early monitoring of anti-PD1 immunotherapy: A proof-of-concept study. Ann Oncol 28: 1996-2001, 2017.

19. Guibert N, Mazieres J, Delaunay M, Casanova A, Farella M, Keller L, Favre G and Pradines A: Monitoring of KRAS-mutated ctDNA to discriminate pseudo-progression from true progression during anti-PD-1 treatment of lung adenocarcinoma. Oncotarget 8: 38056-38060, 2017.

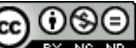

This work is licensed under a Creative Commons Attribution-NonCommercial-NoDerivatives 4.0 International (CC BY-NC-ND 4.0) License. 